\title{
دراسة هحددات وفاة الطفل الأول خلال السنوات الخمس الأولى في الأسر التي تعاني هن هشاكل إنجابية في العاصمة الأردنية عمان
}

إعـــــاد

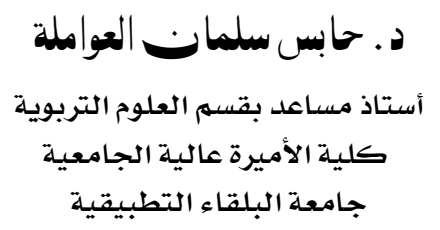

$$
\begin{aligned}
& \text { أ.د. أيمر. سليمار مزاهرة } \\
& \text { أستاذ مشارك- رئيس قسم العلوم التطبيقية }
\end{aligned}
$$

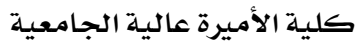

$$
\begin{aligned}
& \text { جامعة البلقاء التطبيقية }
\end{aligned}
$$

مجلة بحوث التربية النوعية - جامعة المنصورة

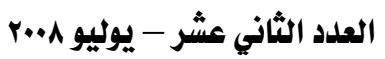




\title{
دراسة همددات وفاة الطفل الأول خلال السنوات الخمس الأولى في الأسر التي تعاني هن هشاكل إبهابية في العاصمة الأردنية عمهان

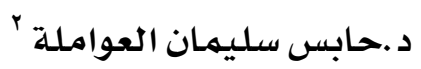 \\ د.أيهن سليهـان مزاهرة '
}

\begin{abstract}
קטגט

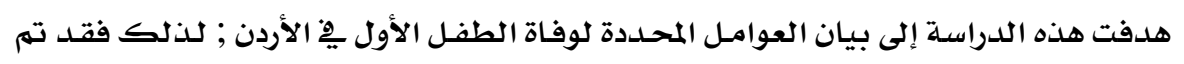

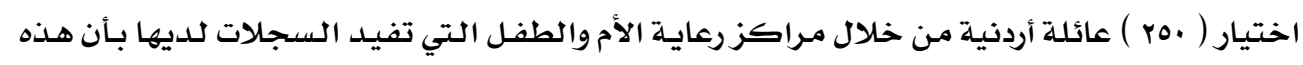

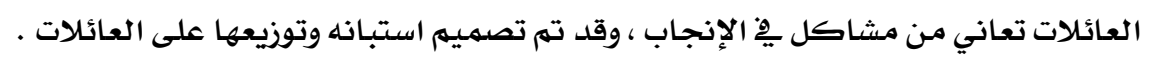

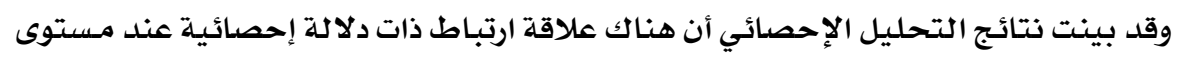

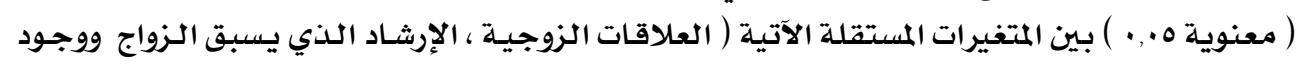

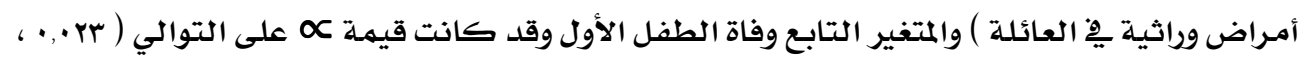

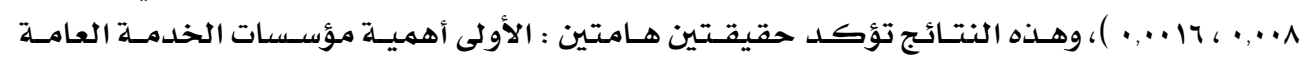

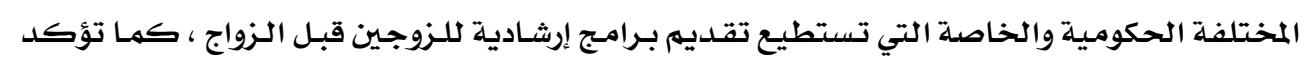

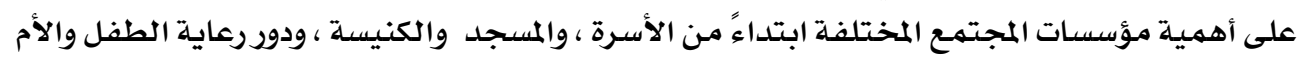

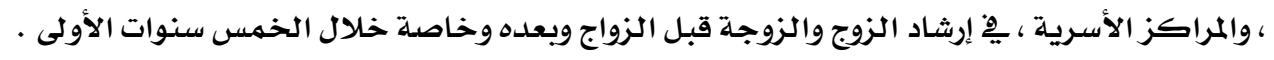

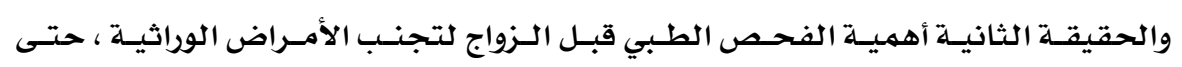

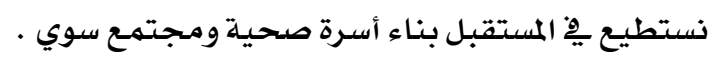

الكلمات المفتاحية : الطفل الاول ، وفاة الطفل ، عمان ، الأردن .

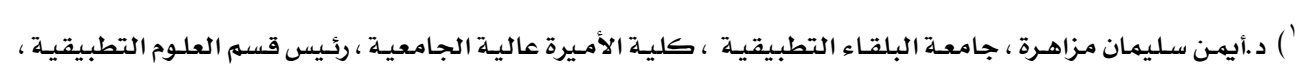

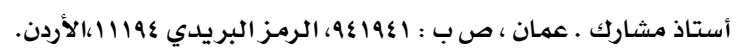

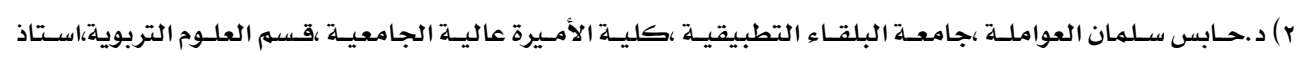

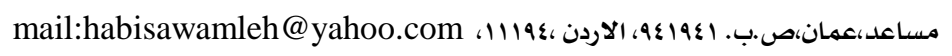




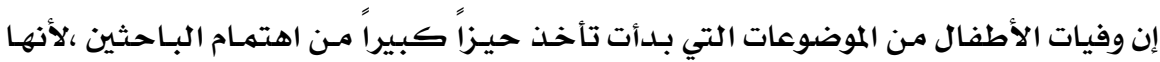

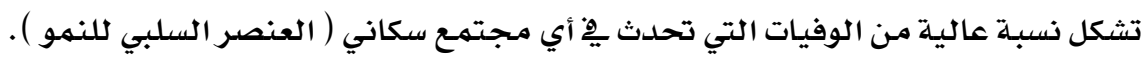
(Hobcraft et.al,1985)

$$
\text { يتوفى دون سن الخامسة (اليونيسف ، ب.... ). }
$$

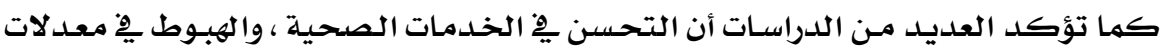

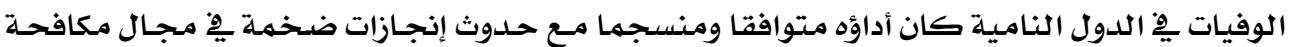

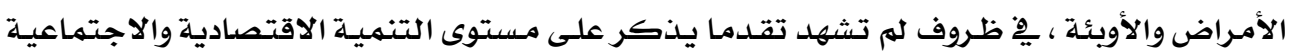

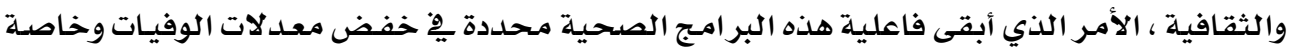

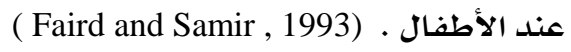
إذن أنّ معدل وفيات الأطفال يعكس بصورة مباشرة الحالة الصحية للسكان ومستوى الرعاية

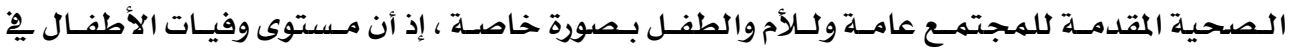

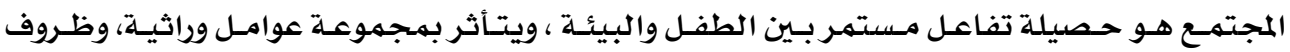

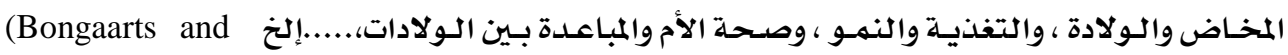

. Potter, 1983)

\section{أهمئية الدراستة :}

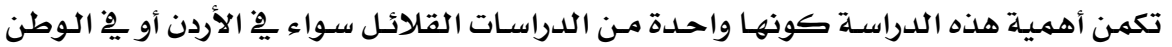

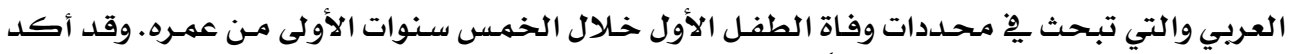

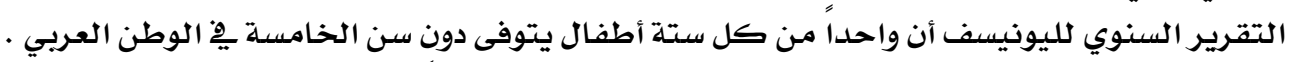

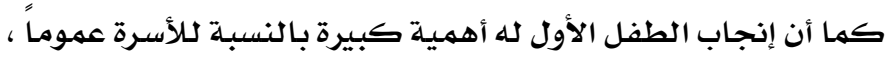

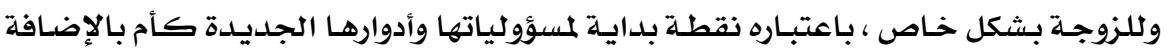

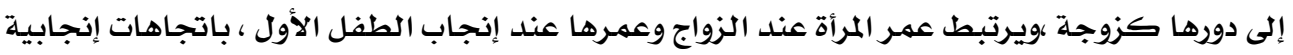

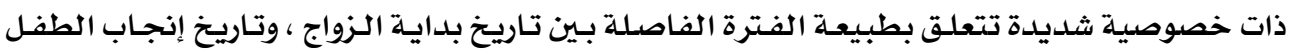

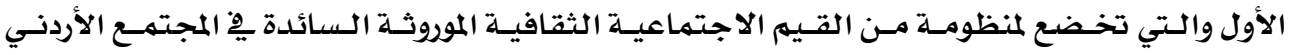

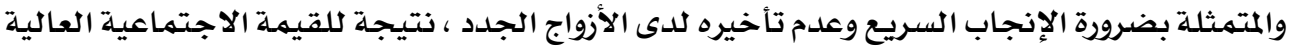

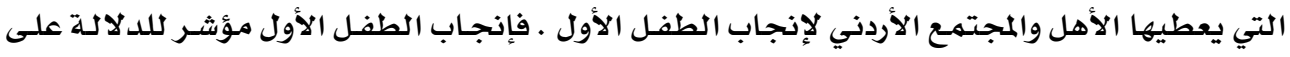

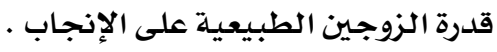

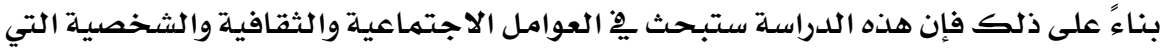

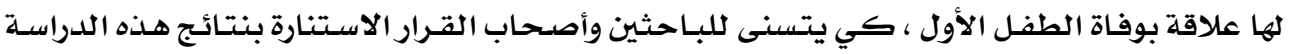

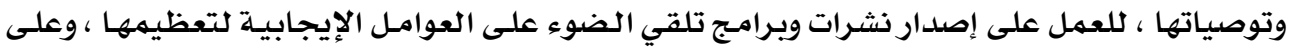

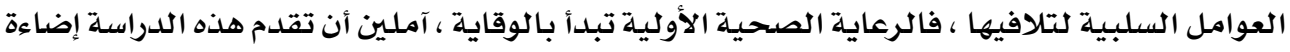




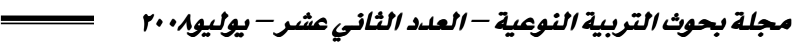

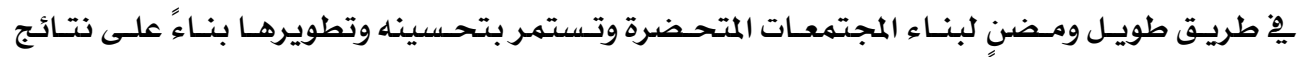

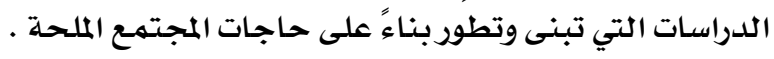

أهداف الدراسة

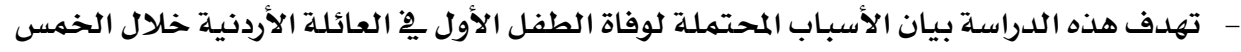

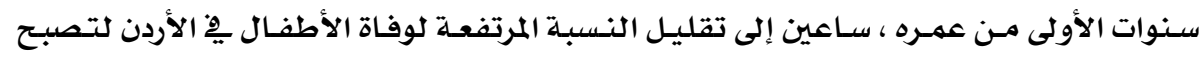

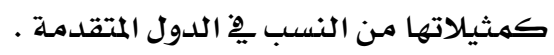

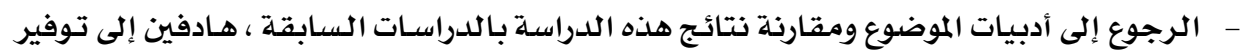

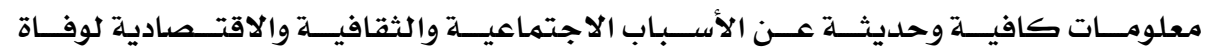

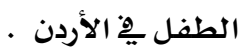

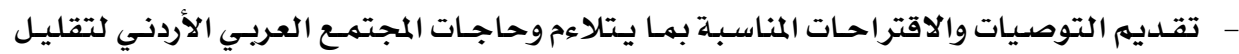

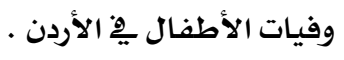

أسـئلة الدراستة:

يمكن تحقيق أهداف الدراسـة من خلال الإجابة عن الأسئلة التالية :

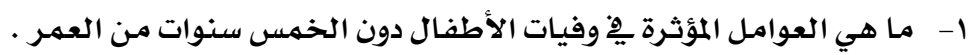

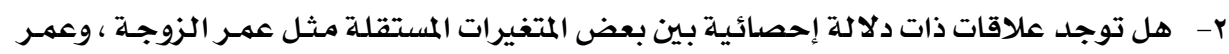

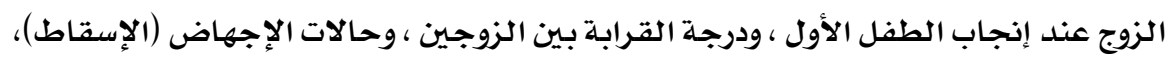

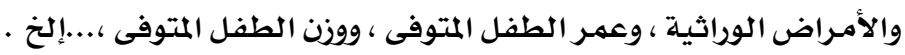

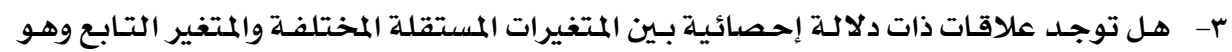

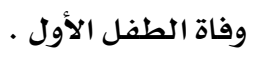

همددات الدراسة: وفاة النفل

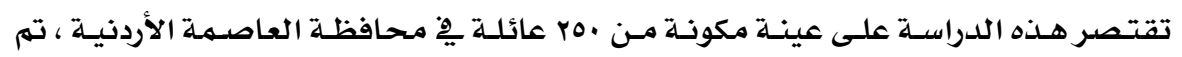

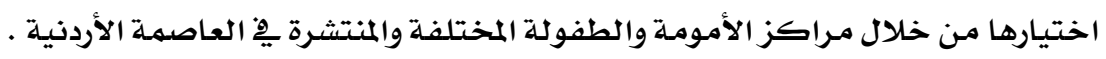

\section{مشكلة الدراسة : مشكالة}

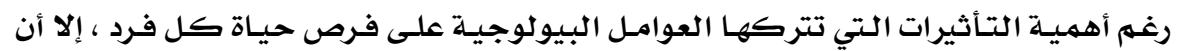

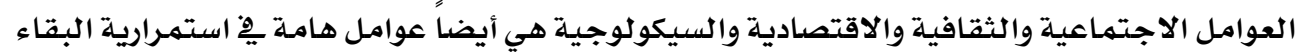

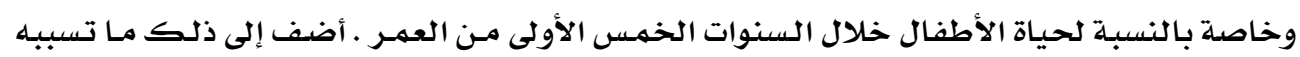

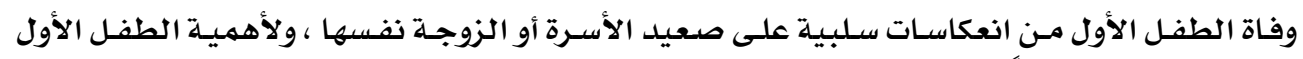

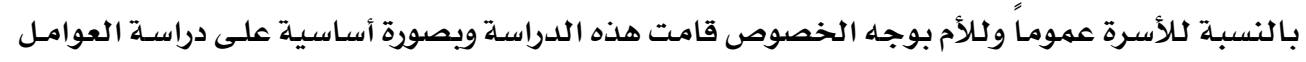

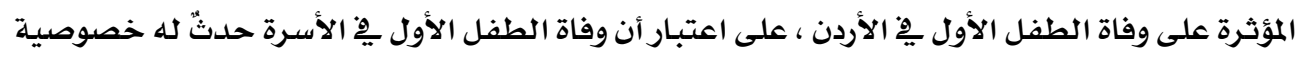

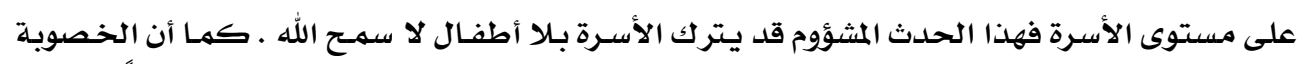

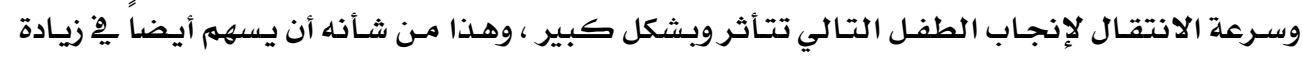


الولادات غير المكتملة ووفيات الأطفال والأمهات ، لذلك كله فقد ارتأينا أن ندرس العوامل المؤثرة ِِّ.

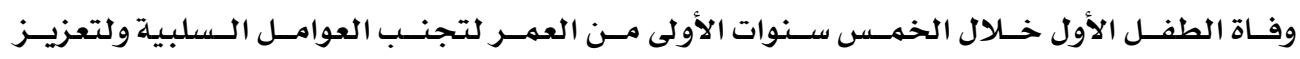
العوامل الإيجابية.

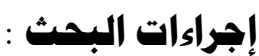

للتعرف على المسببات المحتملة لوفاة الطفل الأول يِّ الأسـرة الأردنيـة يِّ محافظة العاصـمة

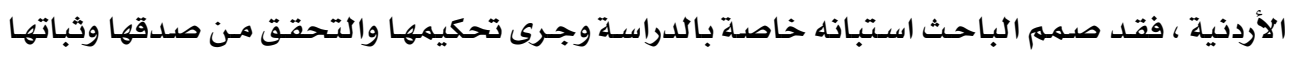

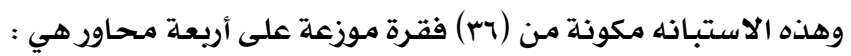

$$
\begin{aligned}
& \text { ا- ا بمعلومات الشخصية للزوج والزوجة . }
\end{aligned}
$$

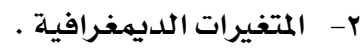

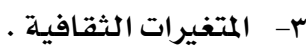

$$
\begin{aligned}
& \text { ع- التغذوي الصحي. }
\end{aligned}
$$

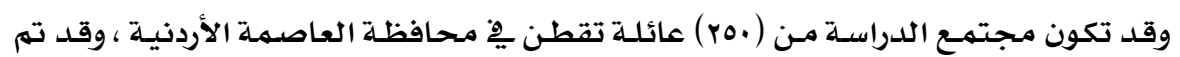

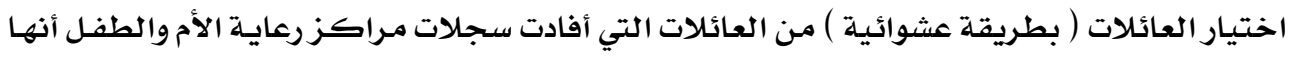

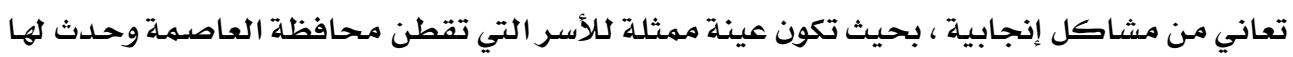

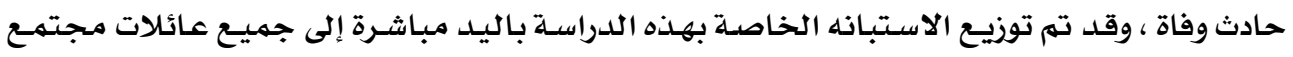

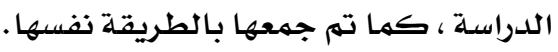

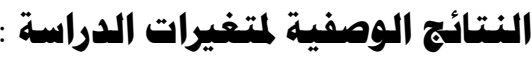

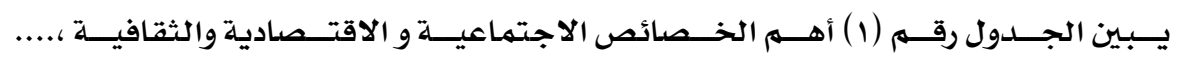
لعينة الدراسة . 


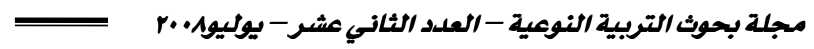

الجدول رقم (1) أهم الخصائص الاجتماعية و الاقتصادية والثقافية ،....لعينة الدراسة

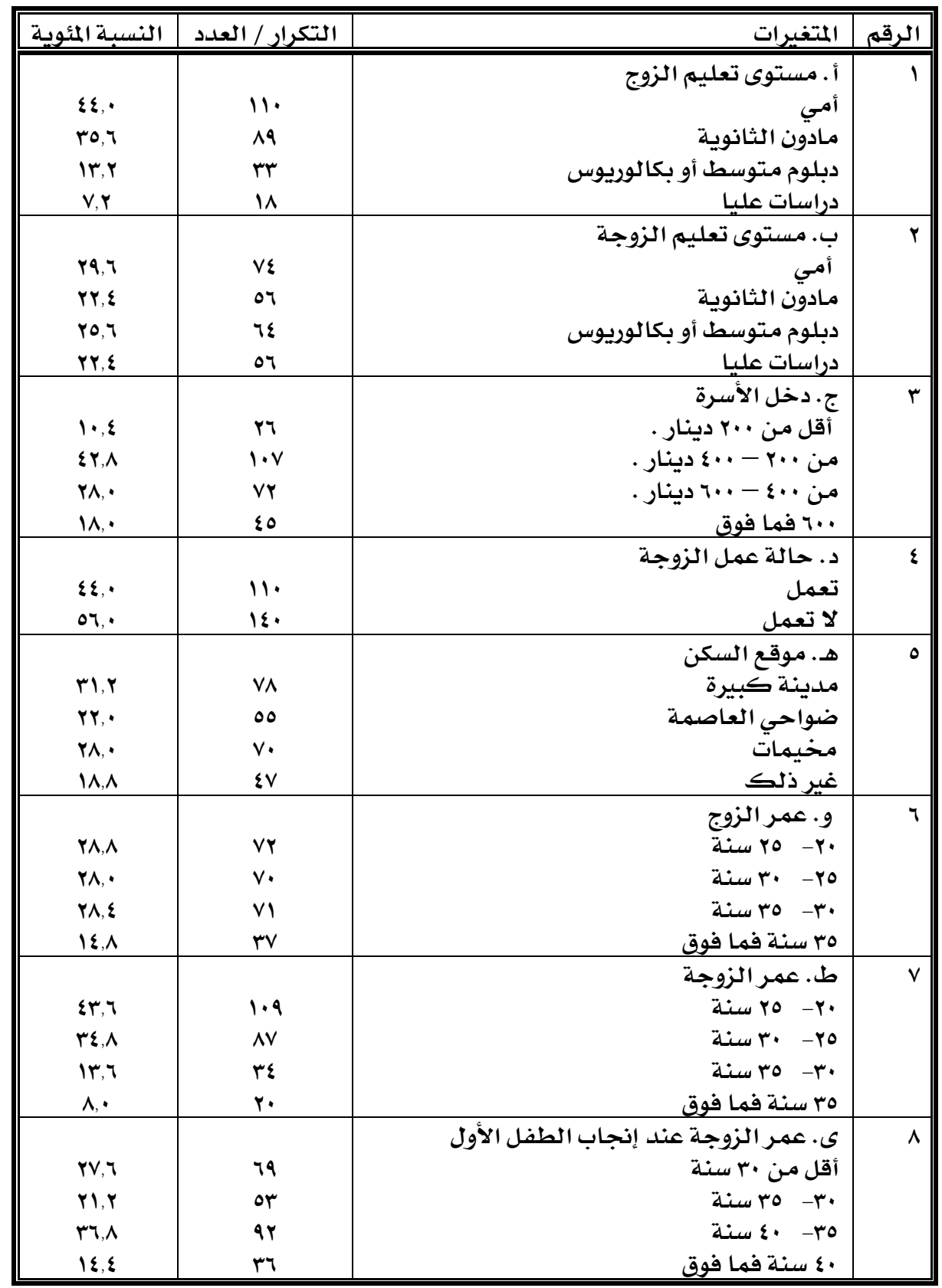


يبين الجدول رقم (r) : التكرارات والنسب المئويـة لإجابات عينة الدراسـة عن أسئلة الاستبانه

\begin{tabular}{|c|c|c|c|}
\hline المئوينة & / التكرار & السؤال & الرقم \\
\hline $\begin{array}{l}T r, \varepsilon \\
r V, T\end{array}$ & $\begin{array}{l}107 \\
98\end{array}$ & هل أنت مـع تحديد النسل ؟ & -1 \\
\hline $\begin{array}{l}\varepsilon r, r \\
r \cdot, \Lambda \\
r \cdot, \cdot \\
r, \cdot\end{array}$ & $\begin{array}{l}1 \cdot 1 \\
V V \\
0 . \\
10\end{array}$ & كه عدد الأطفال الذين ترغب بإنجابهم ؟ & $-r$ \\
\hline $\begin{array}{l}\varepsilon r, 7 \\
07, \varepsilon\end{array}$ & $\begin{array}{l}1.9 \\
1 \leqslant 1\end{array}$ & هل سبق النقاش بين الزوجين بخصوص عدد الأطفال المرغوبين ؟ & r \\
\hline $\begin{array}{l}r q, r \\
7 \cdot, \Lambda\end{array}$ & $\begin{array}{l}91 \\
10 Y\end{array}$ & هل سبق وأن أجريتهم الفحص الطبي قبل الزواج ؟ & $-\varepsilon$ \\
\hline $\begin{array}{l}\varepsilon, \varepsilon \\
19,7 \\
r \varepsilon, \varepsilon \\
r Y, \varepsilon \\
9, Y\end{array}$ & $\begin{array}{l}11 \\
\Sigma 9 \\
\Lambda 7 \\
11 \\
r\end{array}$ & 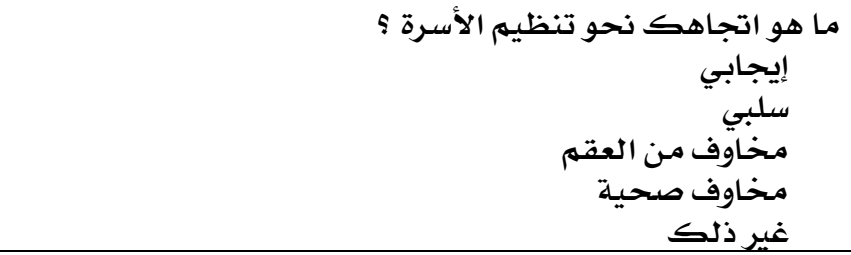 & -0 \\
\hline $\begin{array}{l}\vee 0,7 \\
17, \\
\Lambda, \varepsilon\end{array}$ & $\begin{array}{l}1 \wedge 9 \\
\varepsilon \\
r 1\end{array}$ & 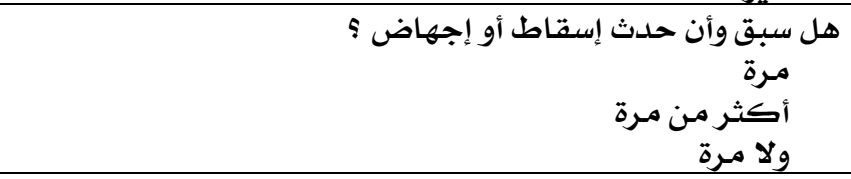 & -7 \\
\hline $\begin{array}{l}r Y, \mathcal{L} \\
r Y, Y \\
r T, \Lambda \\
I V, T\end{array}$ & $\begin{array}{l}07 \\
01 \\
9 Y \\
\varepsilon \varepsilon\end{array}$ & 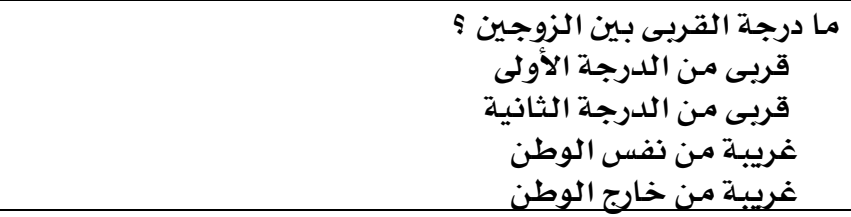 & $-V$ \\
\hline $\begin{array}{l}\varepsilon \cdot \Lambda \\
0 \varepsilon, Y\end{array}$ & $\begin{array}{l}1 \cdot r \\
1 \leqslant \Lambda\end{array}$ & هل توجد أمراض وراثية يِّ العائلة ؟ & $-\wedge$ \\
\hline $\begin{array}{l}\varepsilon, \varepsilon \\
i \Lambda, \\
r 0, r \\
r \wedge, \\
\Lambda, \Lambda \\
0, r\end{array}$ & $\begin{array}{l}11 \\
\varepsilon 0 \\
11 \\
V . \\
r Y \\
1 \varepsilon\end{array}$ & 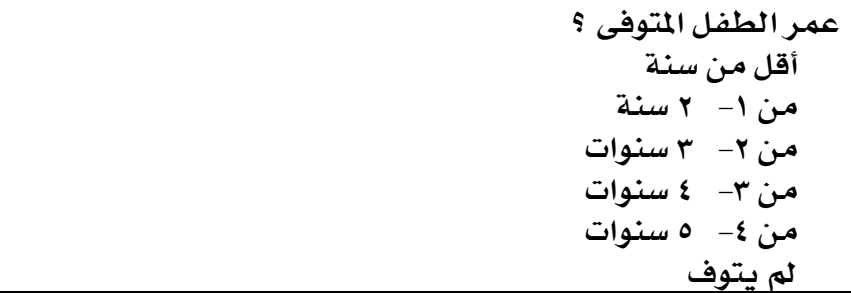 & -9 \\
\hline
\end{tabular}




\begin{tabular}{|c|c|c|c|}
\hline $\begin{array}{c}7 \cdot, \cdot \\
r \varepsilon, \varepsilon \\
0,7\end{array}$ & $\begin{array}{l}10 . \\
17 \\
18\end{array}$ & ما هو جنس الطفل المتوفى ؟ ذأنى & -1 \\
\hline $\begin{array}{l}\Lambda \Lambda, \wedge \\
\|, Y\end{array}$ & $\begin{array}{l}\text { YrY } \\
\text { YA }\end{array}$ & الدين؟ الإسـلام & -11 \\
\hline $\begin{array}{l}01,7 \\
\varepsilon \wedge, \varepsilon\end{array}$ & $\begin{array}{l}\mid r q \\
|r|\end{array}$ & تكرار زواج الزوج؟ & $-1 Y$ \\
\hline $\begin{array}{l}\text { Vד, \& } \\
\text { Yr, }\end{array}$ & $\begin{array}{l}191 \\
09\end{array}$ & هل توفى الطفل الأول يِّ الأسرة ؟ & $-1 \mu$ \\
\hline $\begin{array}{l}V Y, \Lambda \\
7, \Lambda \\
1 \cdot, \cdot \\
1 \cdot, \varepsilon\end{array}$ & $\begin{array}{l}\text { IAY } \\
\text { IV } \\
\text { ro } \\
\text { rA }\end{array}$ & العلاقات الزوجيةت؟ & $-1 \varepsilon$ \\
\hline $\begin{array}{l}\varepsilon \varepsilon, 1 \\
19, Y \\
9, r \\
1 r, Y \\
7, \Lambda \\
7, \Lambda\end{array}$ & $\begin{array}{l}\operatorname{llr} \\
\varepsilon \Lambda \\
r r \\
r r \\
\text { IV } \\
\text { IV }\end{array}$ & مصلدر المعلومات حول تنظيم الأسـرة والصحة والتغذية هو؟ & -10 \\
\hline $\begin{array}{l}r \Lambda, \cdot \\
r r, 7 \\
r r, \cdot \\
17, \varepsilon\end{array}$ & $\begin{array}{l}v . \\
\Delta q \\
\Lambda . \\
\varepsilon 1\end{array}$ & ما هو مصبدر المعلومات المعتمدة حول تنظيم الأسرة ؟ & -17 \\
\hline $\begin{array}{l}r I, T \\
I V, r \\
r T, \\
r I, \cdot \\
I \varepsilon,\end{array}$ & $\begin{array}{l}0 \varepsilon \\
\varepsilon r \\
\text { To } \\
\text { or } \\
\text { ro }\end{array}$ & 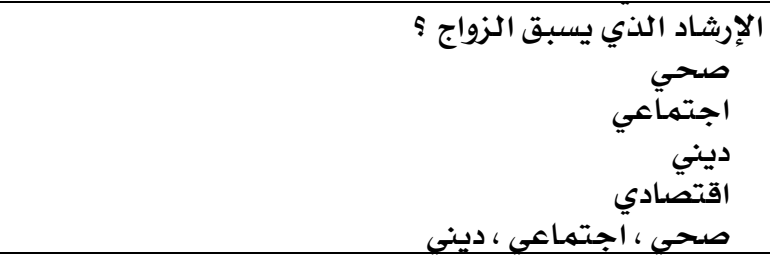 & $-1 V$ \\
\hline $\begin{array}{l}\text { T., } \\
r r, \cdot \\
r r, \cdot \\
r O, r \\
\varepsilon, \cdot\end{array}$ & $\begin{array}{l}10 \\
1 . \\
\text { Nr } \\
\text { rr } \\
1 .\end{array}$ & 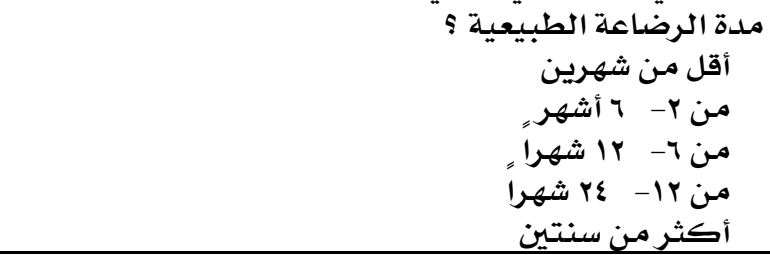 & -11 \\
\hline
\end{tabular}


دراسة محلددات وفاة الطفل الأول خلال السنوات الخمس الأولى

\begin{tabular}{|c|c|c|c|}
\hline $\begin{array}{l}Q Y, \mathcal{Z} \\
V, T\end{array}$ & $\begin{array}{l}\text { rr1 } \\
19\end{array}$ & سبق وأن تم تطعيم أطفال الأسرة ؟ & -19 \\
\hline $\begin{array}{l}T V, T \\
Y r, Y \\
9, Y\end{array}$ & $\begin{array}{l}179 \\
01 \\
r r\end{array}$ & 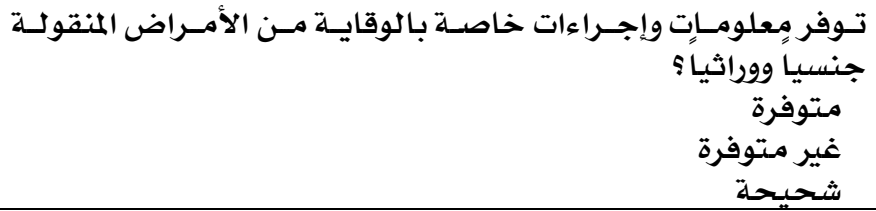 & $-r$. \\
\hline $\begin{array}{l}r \wedge, \varepsilon \\
r \xi, \Lambda \\
r q, \\
10, r \\
0,7\end{array}$ & $\begin{array}{l}\text { VI } \\
\text { Tr } \\
\text { To } \\
\text { rA } \\
\text { IE }\end{array}$ & 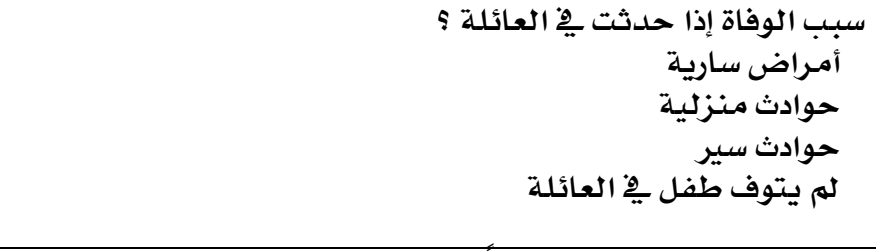 & $-Y_{1}$ \\
\hline $\begin{array}{l}7 \varepsilon, \Lambda \\
r 0, r \\
1 \cdot, \cdot\end{array}$ & $\begin{array}{l}17 r \\
\text { Tr } \\
\text { ro }\end{array}$ & مهارسـة تهارين الريـاضية يوميا ؟ نادرا & $-Y Y$ \\
\hline $\begin{array}{l}\wedge \uparrow, \Lambda \\
1 Y, Y\end{array}$ & $\begin{array}{l}\text { YIV } \\
\text { rr }\end{array}$ & نالاستحمام يوميا؟؟ & Wr \\
\hline $\begin{array}{l}V V, 7 \\
1 \cdot, 1 \\
11,7\end{array}$ & $\begin{array}{l}19 \varepsilon \\
r V \\
r q\end{array}$ & تناول الخهراوات والفواكه يوميا ؟ & $-Y \varepsilon$ \\
\hline $\begin{array}{l}\text { V7, } \\
11,7 \\
1 Y, \varepsilon\end{array}$ & $\begin{array}{l}19 . \\
r 9 \\
r 1\end{array}$ & تناول مشتقات الألبـان يوميأ؟ & $-r_{0}$ \\
\hline $\begin{array}{l}V \varepsilon, \varepsilon \\
1 Y, 7 \\
1 Y, \cdot\end{array}$ & $\begin{array}{l}1 \wedge 7 \\
r \varepsilon \\
r .\end{array}$ & تناول اللحوم والمنتجات الحيوانية ؟ & $-Y T$ \\
\hline $\begin{array}{l}9 \boldsymbol{Y}, \boldsymbol{T} \\
\varepsilon, \cdot \\
r, \varepsilon\end{array}$ & $\begin{array}{l}r r \varepsilon \\
1 . \\
7\end{array}$ & الابتعاد عن الأمـراض السـاريـة ؟ نادرا & $-Y V$ \\
\hline $\begin{array}{l}\varepsilon \cdot \cdot \cdot \\
r \cdot, \cdot \\
r r, r \\
11, r \\
0, r\end{array}$ & $\begin{array}{l}1 \cdots \\
0 . \\
0 \wedge \\
r \wedge \\
1 \varepsilon\end{array}$ & 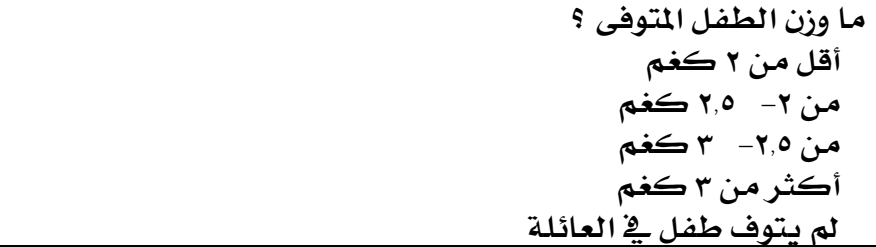 & $-Y \wedge$ \\
\hline
\end{tabular}




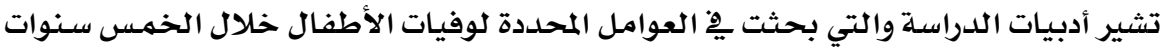

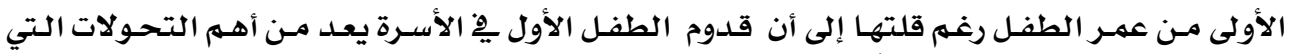

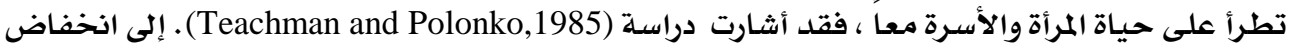

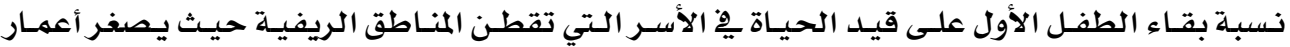

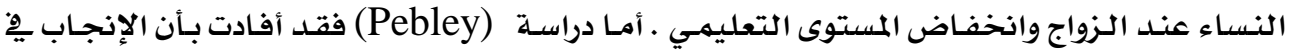

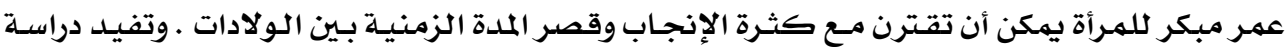
(Miller,1993)

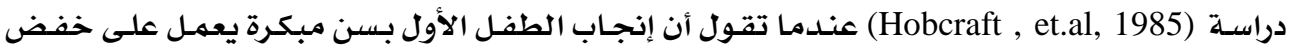
احتمالات بقاء الطفل على قيد الحياة .

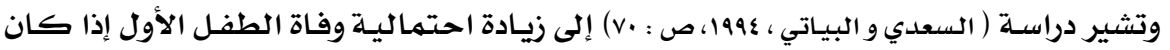

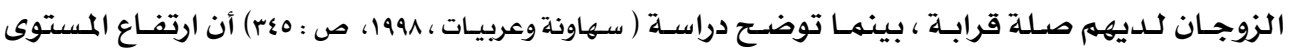

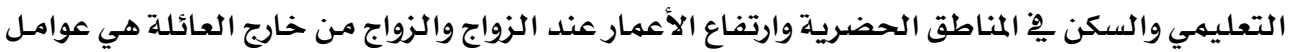
تقلل من احتمالات وفاة الطفل .

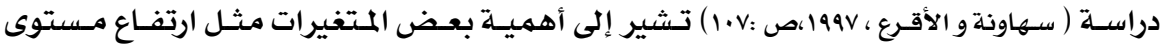

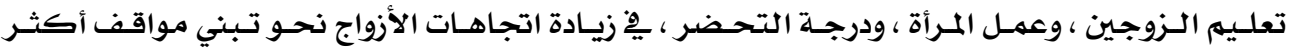

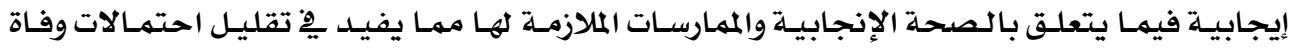

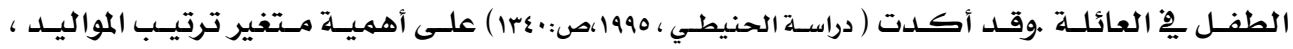

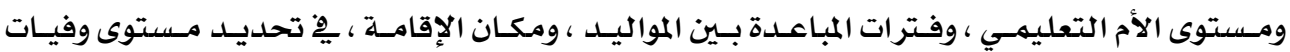
الأطفال الرضع

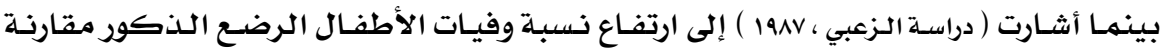

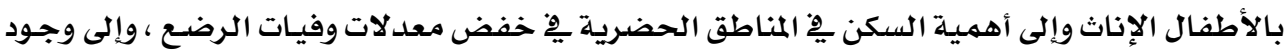

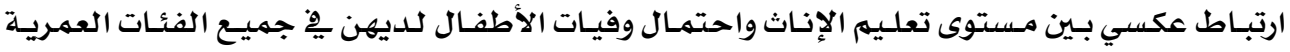

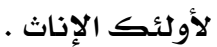

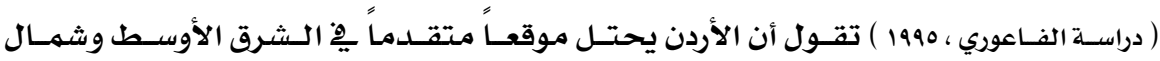

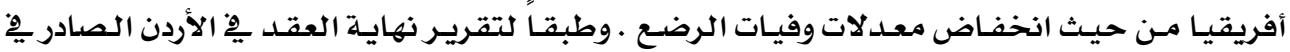

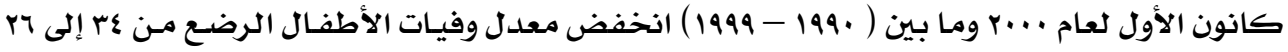

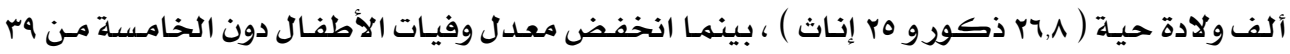

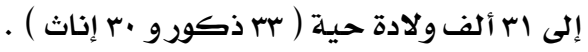

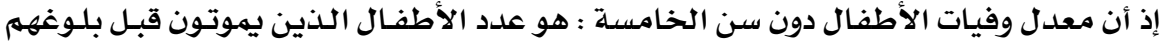

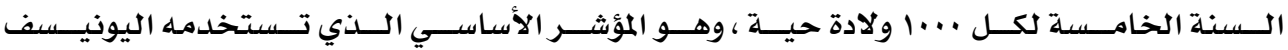
.(Hobcraft,et.al,1985). 


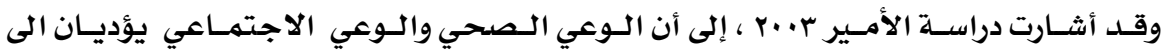

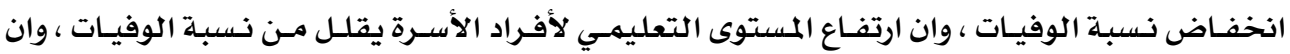

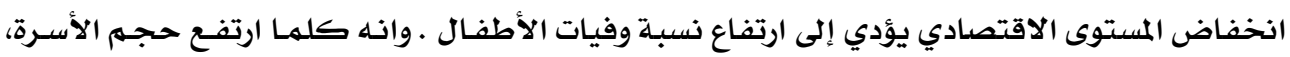

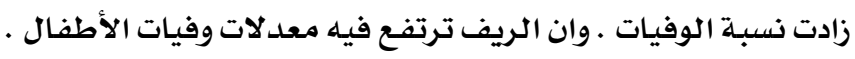

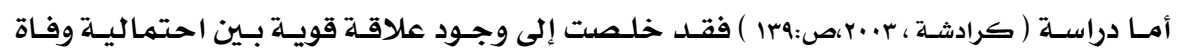

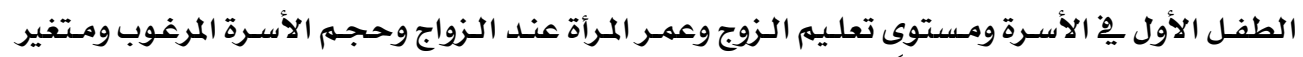

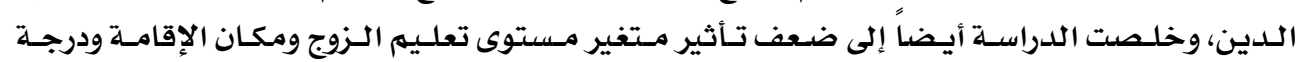

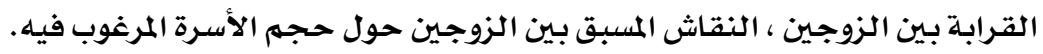

\section{النتائج والمناقشة :}

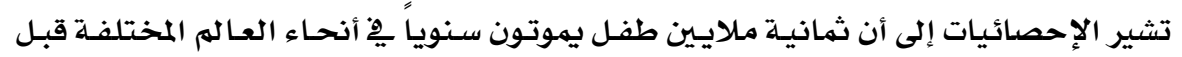

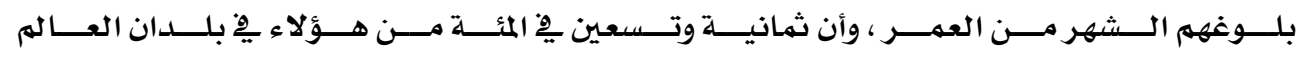

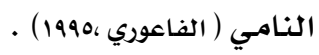

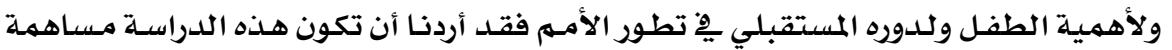

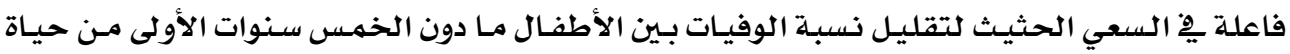

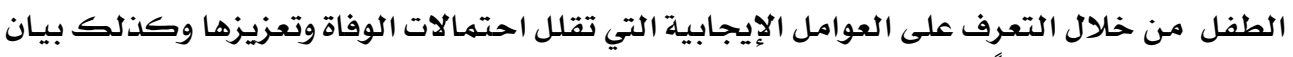

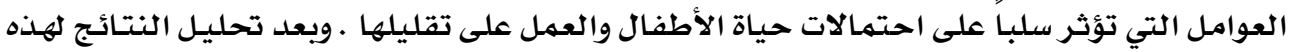

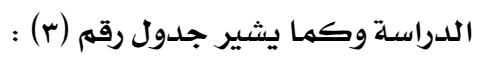

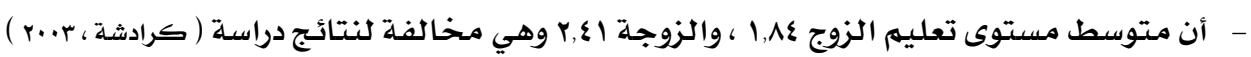

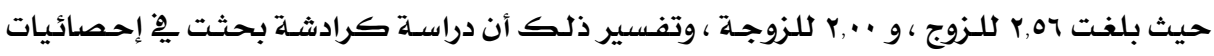

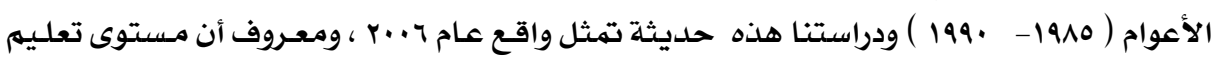

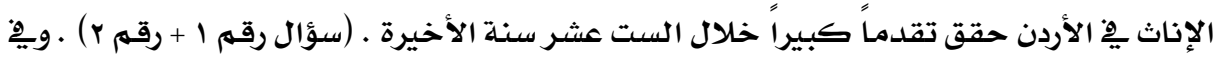

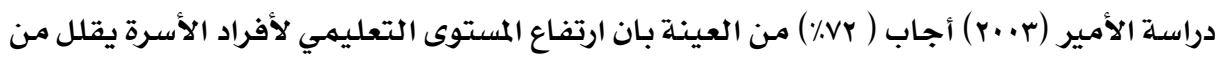

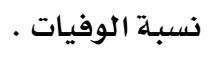

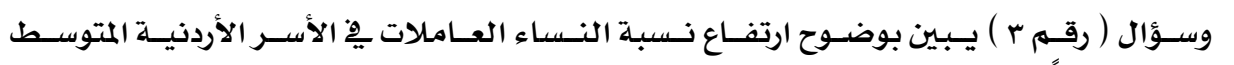

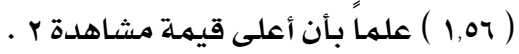

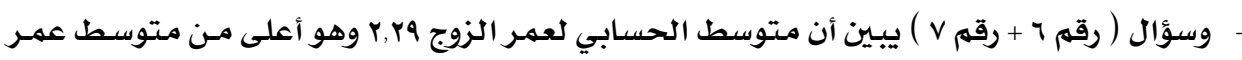

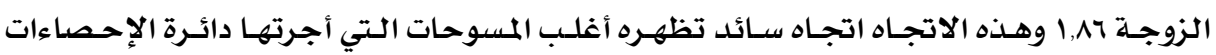

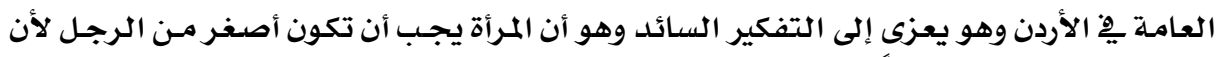
الرجل هو المكلف اقتصادياً بالمرأة من حيث الإلفير الإنفاق والحماية.

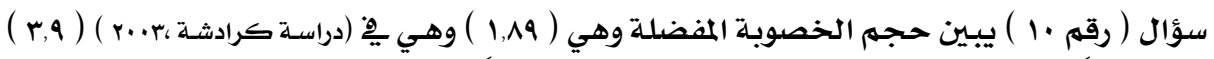

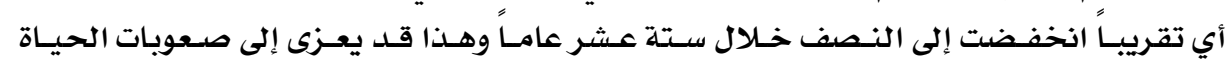




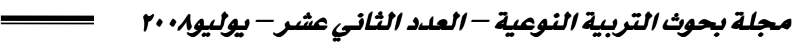

الاقتصادية ومشارككة المرأة يِّ سـوق العمل ، وارتفـاع سـن الزواج ، وتشجيع المجتهـع على بـرامج

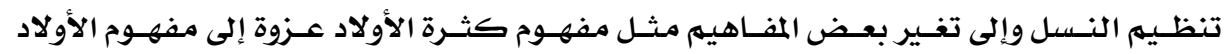

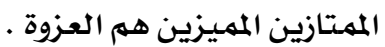

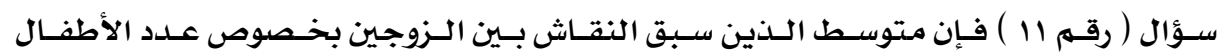

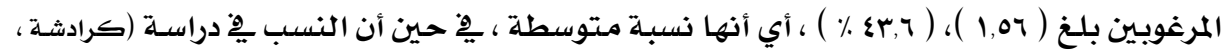

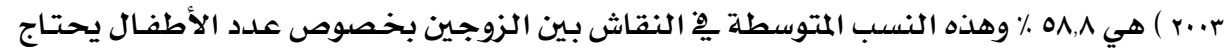

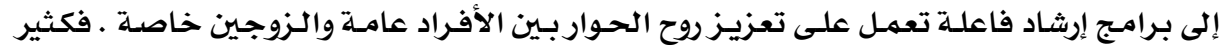
من الأمور تجد طريقا لحلها من خلال الحهاد الحوار البناء.

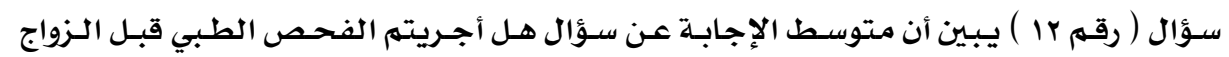

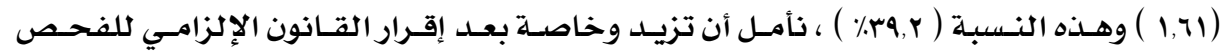

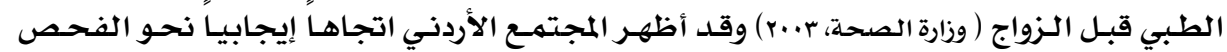

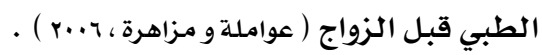

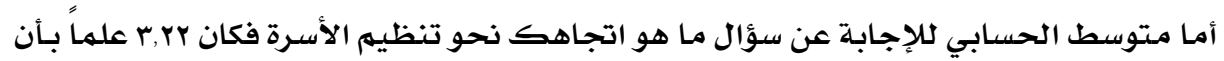

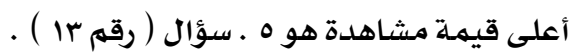

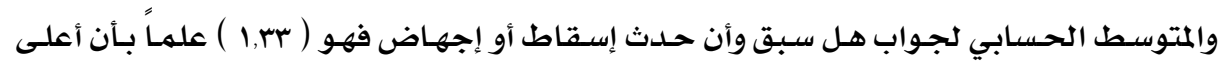

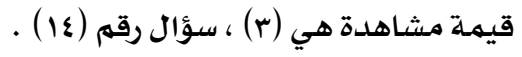

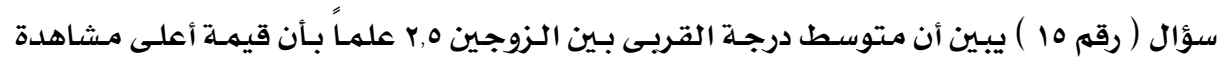

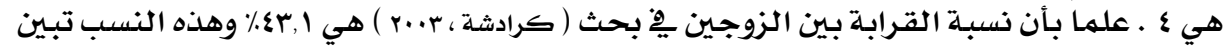

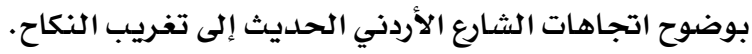

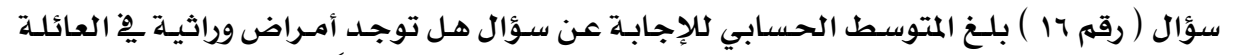

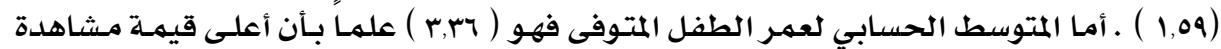

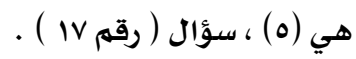

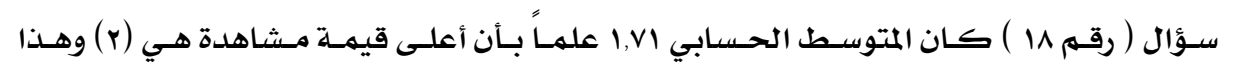

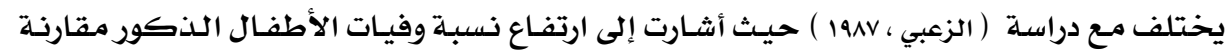

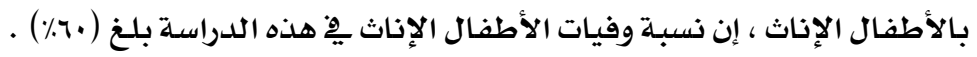

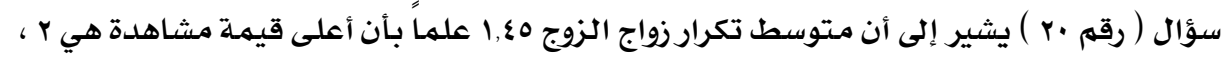

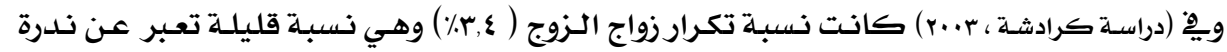

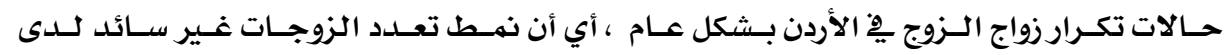

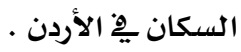




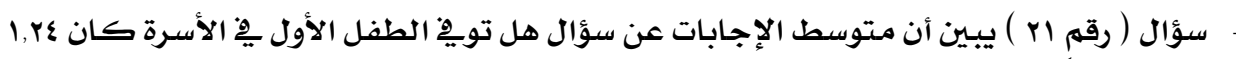

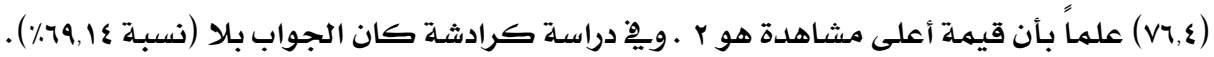

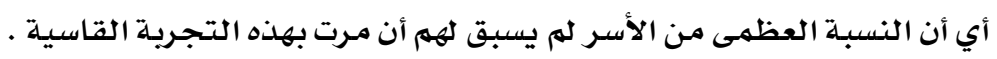

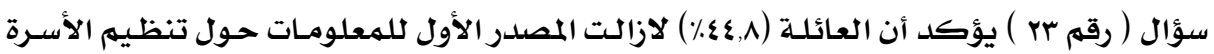

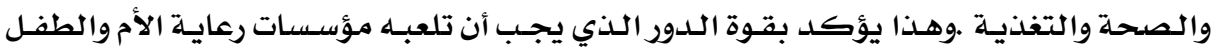

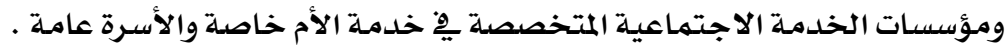

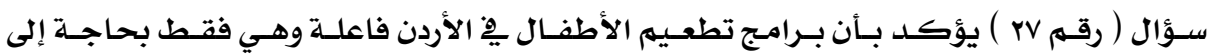

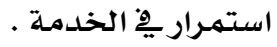

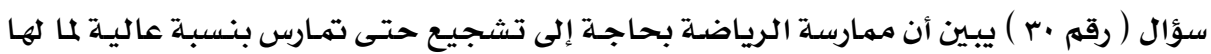

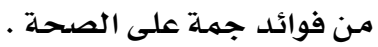

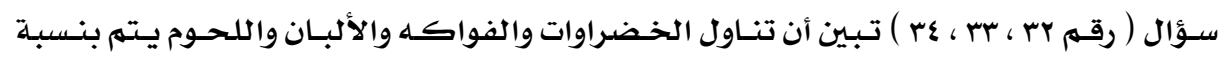
تعتبر قليلة لذلك يجب التركيز على برامج التغذية المتوازنة للطعام والأسرة .

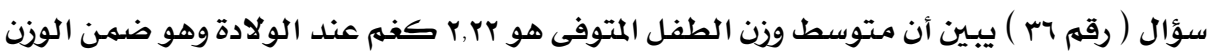

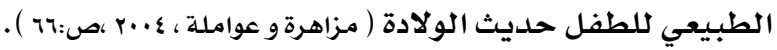

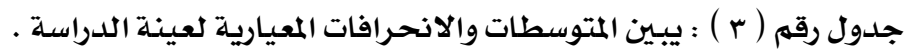

\begin{tabular}{|c|c|c|c|c|c|c|}
\hline قمشاهدة & مشاهدة & المعنوية & الانحرافات & المتوسطات & المتغيرات & المتسلسل \\
\hline$\varepsilon$ & 1 & • &., 910 & $1, \wedge \varepsilon$ & مستوى تعليه الزوج & -1 \\
\hline$\varepsilon$ & 1 & $\cdot, \varepsilon Y 7$ & $1,1 r \varepsilon$ & $r, \varepsilon 1$ & مستوى تعليه الزوجة & $-r$ \\
\hline$\varepsilon$ & 1 & $\cdot, \mathrm{v} 00$ & $\cdot 9 \cdot 0$ & $r, 0 \leqslant$ & 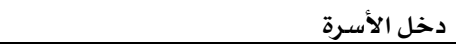 & $-r$ \\
\hline$r$ & 1 & ( & $\cdot, \varepsilon 9 \vee$ & 1,07 & عمل الزوجة & $-\varepsilon$ \\
\hline$\varepsilon$ & 1 & . OY9 & $1,1.9$ & $r, r \varepsilon$ & موقع السكن & -0 \\
\hline$\varepsilon$ & 1 & $\cdot, \varepsilon)$ & $1, \cdot \varepsilon 1$ & $r, Y q$ & عمر الزوج & -7 \\
\hline$\varepsilon$ & 1 & $\cdot, 1 \leqslant 1$ & - 9 qry & $1, \wedge 7$ & عمر الزوجـة & $-v$ \\
\hline$\varepsilon$ & 1 & $\cdot, I V \cdot$ & $1, \cdot r q$ & r,rı & عمـر الزوجة عند إنجاب الطفل الأول & $-\Lambda$ \\
\hline r & 1 & • rیq & $\cdot, \varepsilon \wedge 0$ & I,rی & هل أنت مـع تحديد النسل & -9 \\
\hline$\varepsilon$ & 1 & $\cdot, r \cdot 7$ & $\cdot, 9 Y 9$ & 1,19 & (متوسط عدد الأطفـال الذين ترغب بإنجـابهم & -1 . \\
\hline r & 1 & $\cdot, 810$ & $\cdot, \leqslant 9 \vee$ & 1,07 & 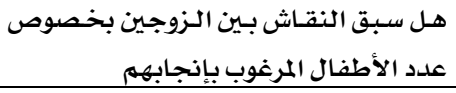 & -11 \\
\hline r & 1 & - val & $\cdot,\{\wedge \uparrow$ & 1,71 & هل سبق وأن أجـريتم الفحص الطبي قبل & $-1 r$ \\
\hline
\end{tabular}




\begin{tabular}{|c|c|c|c|c|c|c|}
\hline مشاهدة & مشاهدة & المعنوية & الانحرافات & المتوسطات & المتغيرات & المتسلسل \\
\hline$\circ$ & 1 & $\cdot, 190$ & $1, \cdots 9$ & $r, r r$ & ما هو اتجاهك نحو تنظيم الأسرة & $-1 r$ \\
\hline r & 1 &., 0 ora & צ & I,rr & 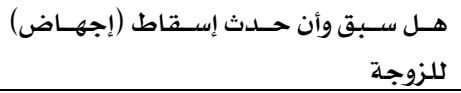 & $-1 \varepsilon$ \\
\hline$\varepsilon$ & 1 & $\cdot, 1 \mathrm{HV}$ & $1, \cdot r V$ & r.o & مـا درجة القربى بين الزوجين & -10 \\
\hline r & 1 & $\$ \cdot, \cdot 17$ & $\cdot, \varepsilon 9 r$ & 1,09 & هل توجـد أمراض وراثية يِّ العائلة & -17 \\
\hline 0 & 1 & $\cdot, r$. & $1, \mid \mathrm{VI}$ & דr,r & عمر الطفل المتوفى & -18 \\
\hline r & 1 & $\cdot$, val & $.07 \varepsilon$ & $1, \times 1$ & جنس الطفل المتوفى & -11 \\
\hline$r$ & 1 & $\cdot, \S 0$. & • & 1,11 & 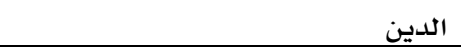 & -19 \\
\hline r & 1 & $\cdot, \wedge 90$ & $\cdot 0.1$ & $1, \varepsilon 0$ & تكرار زواج الزوج & $-r$. \\
\hline r & 1 & - & $\cdot$, \& Y O & $1, r \varepsilon$ & هل تويِ الطفل الأول يِّ الأسرة & $-r_{1}$ \\
\hline$\varepsilon$ & 1 & $* \cdot, \cdots \wedge$ & 1,79 & 1,09 & العلاقات الزوجية & $-r r$ \\
\hline 9 & 1 & $\cdot 90 \mathrm{r}$ & 1,TrY & r.rı & مــــدر المعلومــات حــول تنظــيم الأســرة & r \\
\hline$\varepsilon$ & 1 & $\cdot,\{09$ & $1, \cdot 71$ & r,rv & 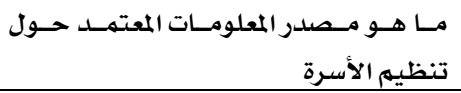 & $-r \varepsilon$ \\
\hline$\varepsilon$ & 1 & מr & $1, r \varepsilon r$ & $r, \wedge 9$ & الإرشـاد الذي يسبق الزواج & $-r_{0}$ \\
\hline$\circ$ & 1 & $\cdot, 0 \cdot 1$ & $\cdot 9 \wedge r$ & $r, \wedge 9$ & مدة الرضاعة الطبيعية & $-r$ \\
\hline$r$ & 1 & $\cdot, 10 \mathrm{~V}$ & $\cdot$, Y^T & $1, \cdot 1$ & سبق وأن تم تطعيم الأسرة & $-r v$ \\
\hline$r$ & 1 & $\cdot, 1 \leqslant r$ &., 700 & $1, \varepsilon r$ & مـ تـوفر معلومـات وإجـراءات خـاصسة للوقايـة & $-r \wedge$ \\
\hline$\varepsilon$ & 1 & • ror & $1, r \cdot q$ & $r, \S 0$ & سبب الوفاة & $-r q$ \\
\hline$r$ & 1 & 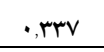 & $\cdot, \mathrm{TV} \cdot$ & $1, \leqslant 0$ & ممارسـة تمارين رياضية يومياً & $-r$. \\
\hline$r$ & 1 & .097 & 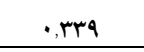 & $1,1 \mu$ & الاستحمام يومياً & $-r_{1}$ \\
\hline$r$ & 1 & $\cdot, \wedge r \mathrm{~V}$ & $\cdot, \mathrm{TVV}$ & $1, r \varepsilon$ & تناول الخضراوات والفواكه يومياً & $-r r$ \\
\hline$r$ & 1 & $\cdot, \mathrm{V} \varepsilon \varepsilon$ & $\cdot, 79 \varepsilon$ & 1, & تناول مشتقات الألبـان يومياً & 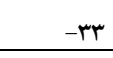 \\
\hline r & 1 & $\cdot, \Lambda T 1$ & $\cdot, 79$. & I, rی & تناول اللحوم والمنتججات الحيوانية يومياً & $-r \varepsilon$ \\
\hline$r$ & 1 & $\cdot, \pi r r$ & . r०9 & $1, \cdot 9$ & الابتعاد عن الأمـراض السـارية & -ro \\
\hline$\varepsilon$ & 1 & $\therefore, 9 \vee 9$ & $1, r \leqslant 1$ & r,rY & وزن الطفل المتوفى عند الميلاد & 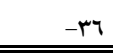 \\
\hline
\end{tabular}

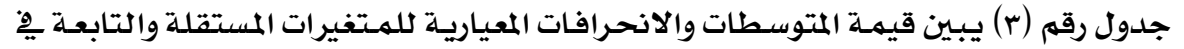

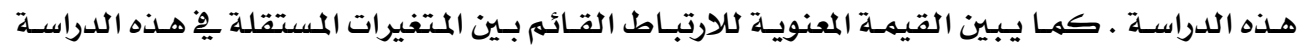

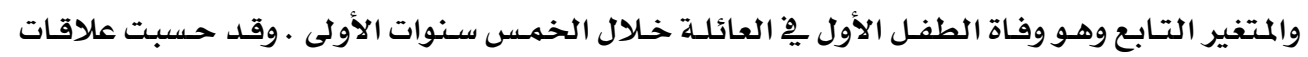




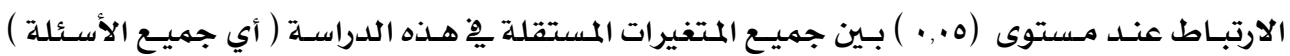

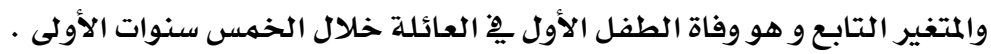

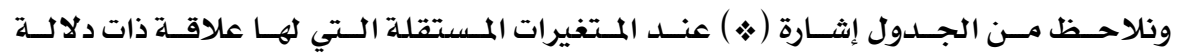

إحصائية وهي :

ا - علاقة ذات دلالة إحصائية بين وفاة الطفل الأول خلا الخمس سنوات الأولى مع متغير وجـود

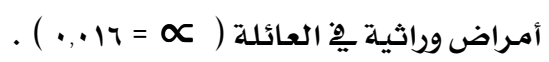

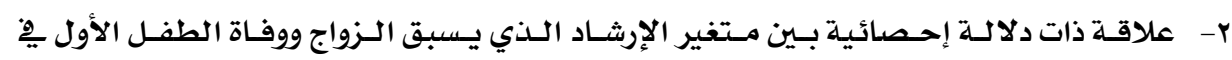

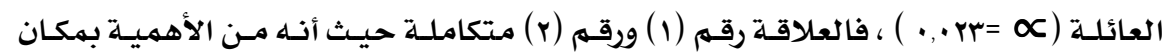

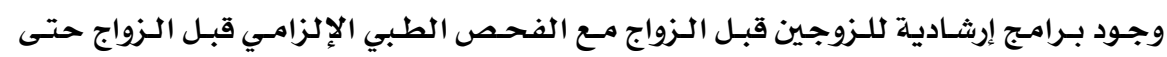

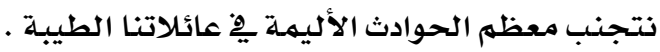

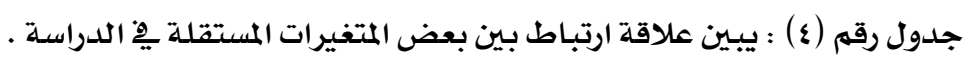

\begin{tabular}{|c|c|c|c|c|c|c|c|c|}
\hline وزن الطفل & الطفل المتوفى & |ممر الطفل & الأمراض العاثية & $\begin{array}{c}\text { (إسقاط) حدوث) } \\
\text { الإجهاض }\end{array}$ & القرابة & |عمر الزوجة الطفاب & الزوجة & \\
\hline$\cdot, 79 V$ & $\cdot r \leqslant r$ & $\cdot, r V Y$ & $\cdot r \cdot r$ & $\cdot, \mathrm{V} \wedge \mathrm{O}$ & • YNT & אדצו, & - & عمر الزوجة \\
\hline$\cdot r v$. & $\cdot, \varepsilon q \mu$ & $\cdot, 179$ & $\cdot, \Lambda \vee \wedge$ & $\cdot, \bullet \wedge$ & $\cdot .999$ & - & • & | عمـر الزوجة عند الطفل الأول \\
\hline$\cdot, 119$ &., 909 & $\cdot, 9 \cdot r$ & $\cdot .70$ &. .91 & - &., 99 & • YAT & درجة القرابة \\
\hline$\cdot, \Sigma 90$ &., 91 & $\cdot, \varepsilon \vee 9$ & $\cdot, 1 Y 0$ & - &., 91 & $\cdot \cdot \cdot \wedge 1$ & $\cdot, \vee \vee \wedge ०$ & إسقاط ( إجهاض) \\
\hline., $09 \varepsilon$ & • & $\cdot, 9 \xi$. & - &., 1 ro &., .70 & $\cdot, \wedge \vee \wedge$ & $\cdot, r \cdot r$ & الأمراض الوراثية \\
\hline$\leftrightarrow \cdot, \cdots$ & $\leftrightarrow \cdot, \ldots$ & - & $\cdot 9 \xi$. & $\cdot, \varepsilon \vee 9$ & $\cdot, 9 \cdot r$ & $\cdot, 179$ & , rVY & عمر الطفل المتوفى \\
\hline$* \cdot, \cdots$ & - & $* \cdot, \cdots$ & •, arr &., .91 &., 909 & • & $\cdot r \leqslant r$ & جنس الطفل \\
\hline - & $* \cdot \cdots$ & $* \cdot \cdots$ & $\cdot .09 \xi$ & $\cdot, \$ 90$ & $\cdot, 119$ & $\cdot r v$. & $\cdot, 79 V$ & وزن الطفل المتوفى \\
\hline
\end{tabular}

• علاقات الارتباط معنوية على مستوى دلالة ا... ( أ>> 1.. ) . .

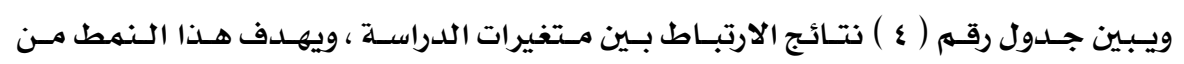

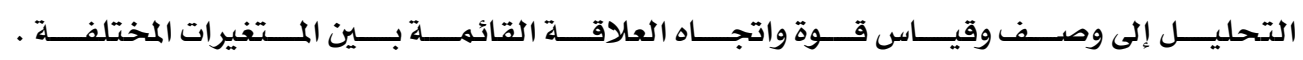

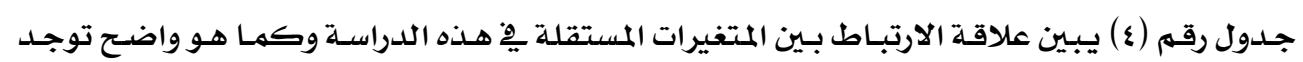

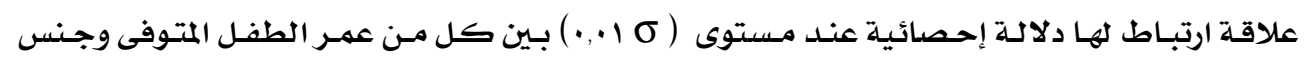

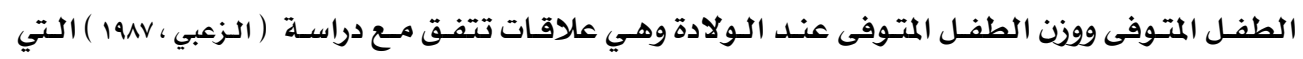

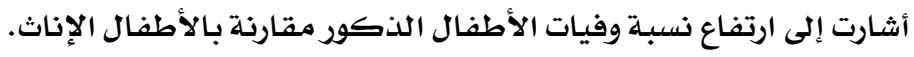


مجلة بحوث التربية النوعية - العلد الثانسي عشر - يوليوب.•r م

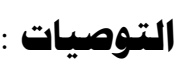

يهكن التقليل من حدوث وفاة للأطفال مـن خلال الإجـراءات الآتية:

الاستمـرار يِ رفع مستوى الخدمات الصحية المقدمـة وتقديه المعلومات خاصدة للأمهات الأميات

واللواتي يقطن يِّ المناطق النائيـة .

- رفع مستوى وعي المرأة الصحي والإنجابي حول خدمات الأمومـة والطفولة .

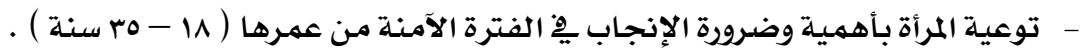

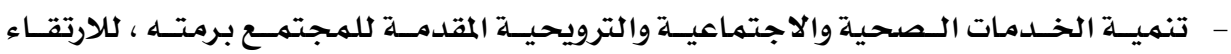

بالمستوى الثقايف والصحي والاجتماعي للمهتهمع عامـة والمرأة خاصسة .

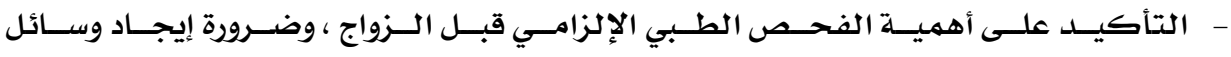
التوعية الناجحة .

- ضـرورة تعميـق الـوعي الاجتمهـاعي عنـــ الأسـرة ، لان ذلـك يحـافظ على الأطفـال ، ويقلـل مـن مسببـات الوفاة . 


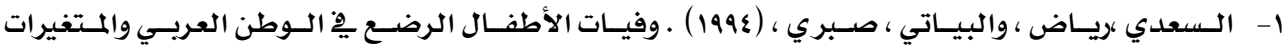

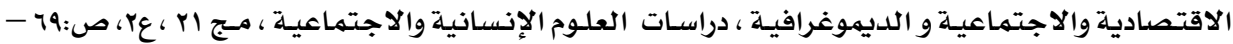

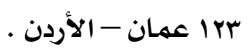

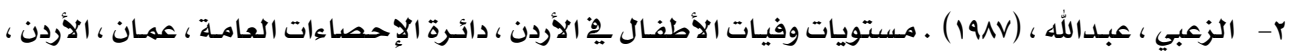
دراسـة غير منشورة .

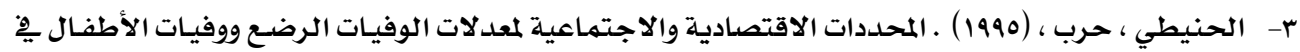

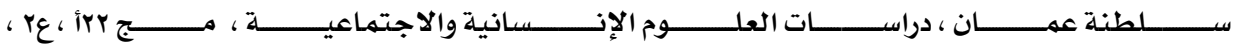

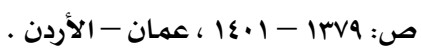

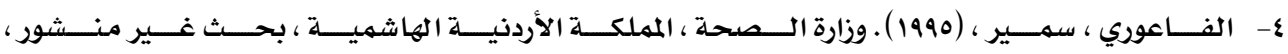

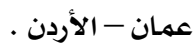

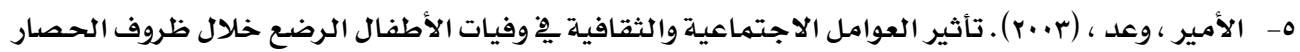

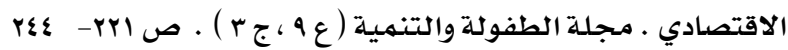

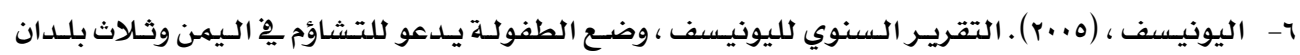

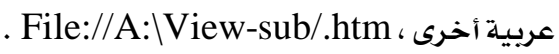

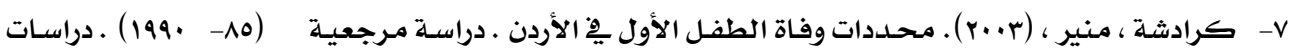

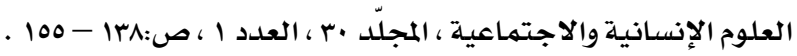

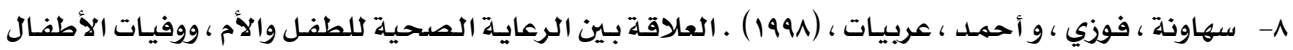

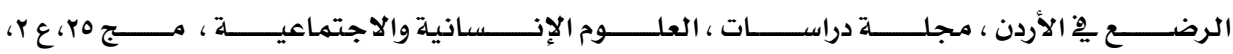

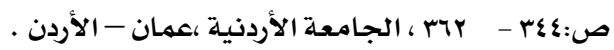

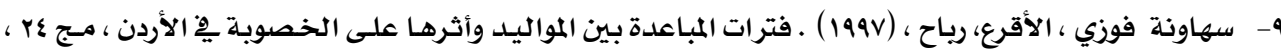

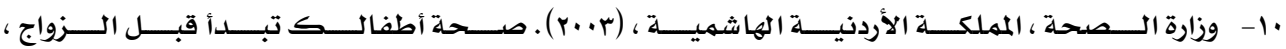

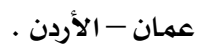

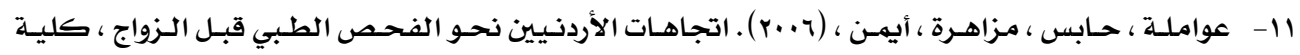

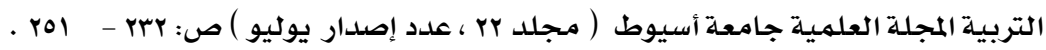

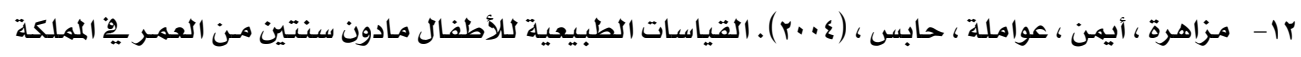

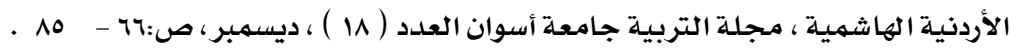

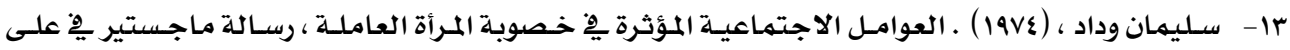

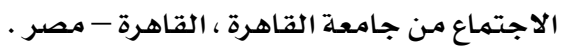

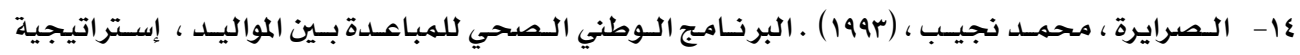

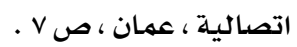




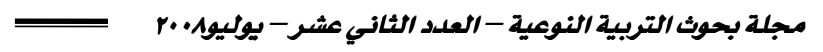

15-Bongaarts . J., and R.Potter. (1983) . Fertility , Biology and Behavior : An Analysis of the Proximate Determinates, New York :Academic Press.

16-Farid, Samir . (1993) . Family Planning Health and Family well-being in the Arab World :Paper presented at the Arab population conference in Amman , Jordan.

17-Hobcraft . J.w., and J.w.McDonald, and Rutstein . (1985) . Demographic Determinants of Infant and Early Child Mortality : A Comparative Analysis . Populalion Studies, No.39.

18-Miller , J. (1993) . Birth Out Comes by mother's Age at First Birth in the Philippines International Family Planning perspectives .

19-Teachman , J.D., and K.Polonko . (1985) . Timing of the Transition to Parenthood : a multidimensional Birth interval Approach: Journal of Marriage and the Family (47):867-879 .

20-Pebley A.R and Alsara. (1986) . Birth Spacing and Child Survival , Interactions Family Planing perspective 12 (3). 
دراسة محددات وفاة الطفل الأول خلال السنوات الخمس الأولى

\title{
Abstract
}

\section{A study on the factors of first born's death during the first five years of marriage in families who live in Amman, The capital of Jordan, that suffer from maternity problems.}

\author{
Dr.Ayman S.Mazahreh * \\ Dr. Habes S. Al-awamleh"*
}

This study is intended to figure out the factors of first born's death in Jordan. The sample consisted of 250 Jordanian families taken from the children for families that have maternity problems .

A questionnaire was designed and gives to these families .

The statistical analyses result show that there is a relationship with statistical indications at the level 0.05 between the independent variables( marriage relations, pre-marriage counseling and hereditary diseases ) and the variable of first born's death .The $\alpha$ value was an the following : ( 0.023 $-0.008-0.0016)$, these results emphasize on the two important issues, the first one is the importance of different public utility, whether it is private or governmental ones that give pre-marriage counciling schedules to couples before getting married. Also, it insists on the importance os social foundations beginning from family centers in giving pre-marriage counciling for couple before and after getting married specially in the first five years of marriage .

The second one is, the importance of pre-marriage medical tests to avoid hereditary diseases in order to be able to have healthy families and societies.

Keywords: $\quad$ First born , born's death , Amman , Jordan .

'Dr.Ayman S.Mazahreh, Al-Balqa University, Princess Alia' University College .Amman 941941,Jordan 11194.

" Dr.Habes S.Awamleh , Al-Balqa University ,Princess Alia University College , Educational Psychology Department , Amman , 941941, Jordan 11194 Email: habisawamleh@yahoo.com 